\title{
Applications of Polymers in Perovskite Solar Cells: A Review
}

\author{
Subhasis Roy* and Shyamal Datta
}

Department of Chemical Engineering, University of calcutta, India

ISSN : 2688-8394

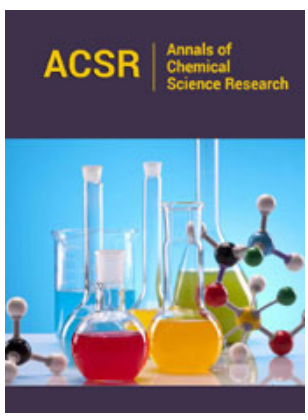

*Corresponding author: Subhasis Roy, Department of Chemical Engineering, University of calcutta, India

Submission: 韭 April 23, 2020

Published: 眥May 28, 2020

Volume 2 - Issue 2

How to cite this article: Subhasis Roy Shyamal Datta. Applications of Polymers in Perovskite Solar Cells: A Review. Ann Chem Sci Res. 2(2). ACSR. 000531.2020. DOI: 10.31031/ACSR.2020.02.000531

Copyright@ Subhasis Roy, This article is distributed under the terms of the Creative Commons Attribution 4.0 International License, which permits unrestricted use and redistribution provided that the original author and source are credited.

\section{Abstract}

Emerging technology and recent research activity help perovskite solar cells to cross with a notable $22 \%$ efficiency. Rapid research and development in organic photovoltaics (OPVs) and light-emitting diodes (OLEDs) leads to optimize the efficiency further. Device efficiency and stability largely depend on components and the device structure of the solar cell. The aim of this report is to review the different strategies employed polymer as an electron transport material (ETM), hole transporting material (HTM) or as a templating agent to enhance the performance, stability, and durability of the perovskite solar cell.

Keywords: Perovskite; Polymers; Solar cells; Efficiency

\section{Introduction}

Modern technology has a growing interest in the application of polymer as a flexible substrate in Dye-sensitized solar cells (DSSC), film-forming agents of the working electrode, platinum-free counter electrodes, and semi solid-state electrolyte. Uses of polymer in perovskite material lead to crystallization processes and work as an additive to adjust the nucleation. These polymers are generally used as an electron transfer material, hole transfer material or interface layer and work like to reduce the recombination rate and improves the separation efficiency of the charge carriers [1]. Polymers are often used as buffer layers or donor layers in inorganic solar cells. Some polymer nanostructured are used to enhance device performance [2,3]. Some low bandgap polymer materials are used as a hole transporting material in perovskite solar cells (PSC) to optimize efficiency as well as prevent cell degradation. Researchers are using a polymer-perovskite composite material to fabricate hybrid structures that are widely used in perovskite-based light-emitting diode (LED). Polymers encapsulated perovskite solar cell has a comparative longer lifetime and less degradation of materials than without encapsulation [4]. The light absorption of Perovskite layer is less impacted by the polymer coating. Though polymers have a lot of advantages for uses in perovskite solar cell some issues like thermal stability in high temperature, water permeability through polymer layer and adhesive property of thin layer is challenging and lot of research work is going on to address the challenges [5]. Recent trends of polymers application in perovskite-based solar cells and its achievements are reviewed and discussed in this article. Moreover, the practical challenges of polymer layered perovskite solar cell and implementation techniques are addressed.

\section{Different Applications of Polymer on Perovskite Solar Cells}

A. Polymer-based photovoltaic technology like Dye-sensitized solar cells (DSSC), perovskite solar cell (PSC), Organic Photovoltaic Solar Cells (OPV) are the key interest nowadays. In DSSC polymers work like a film-forming agent of photoanode and best suitable for the flexible substrate. The platinum-free counter electrode can be fabricated by conductive polymers as Conductive polymers exhibit high catalytic activity which can be ideal for perovskite solar cells [6]. Polymers are widely used in DSSC as a semi solidstate electrolyte.

B. Polymers are widely used in PSC as it facilitates the nucleation process which regulates the crystallization of perovskite films and enhances cell performance and 
stability. Polymers are often chosen as a hole transport material due to significant high hole mobility [7].

C. Light-harvesting efficiency and performances of devices are largely influenced by the polymer addition in OPV. Improvement of the efficiency of the OPV is possible by tuning with a novel polymer having narrow bandgap and appropriate energy level alignment [8]. Besides this, the application of polymer is well promising for the tandem OPV fabricated with a tunable spectrum absorption range.

D. Various OPV and Organic Light-Emitting Diodes (OLED) research facilitates in the perovskite research area and helped by adding polymers which were already been used in OPV or OLED. Previous researches have helped a lot in developing perovskite device as polymers provide a various solution like providing quality charge transport and absorber [9].

E. Despite having high-efficiency perovskite solar modules are still not commercialized. Instability is one of the major drawbacks. With more than 2 decades OPV cells have witnessed lots of improvements and evolution. Operational feasibility and environmental stability were thoroughly studied, however, PSC has very little statistical data to study and worked on. To overcome the instability problem, polymers could serve as a templating agent, acts as a barrier and resist the cell from moisture [10].

F. Heterojunction layer of P3HT: PCBM (poly(3hexylthiophene): [6,6]-phenyl C61-butyric acid methyl ester) works as a control scheme for the past few years due to its predictable performance and characteristics in OPV. Adding polymers to lead-based halide perovskite leads to increase chemical stability; however, their stability mechanism is poorly understood especially for hybrid materials. Several perovskite hybrid models for solar cells have been compared for stability enhancement, structural and spectroscopic analysis and it was found that perovskite crystallite sizes decrease with the addition of polymers (polyethylene glycol, polyethyleneimine, poly(acrylic acid) and polyvinylpyrrolidone) [11].

Some Polymers used in Recent Research in Perovskite Solar Cell

\section{Polyanilines (PANI)}

PANI has high conductivity and used as a hole transporting material. PANI nanoparticles have between $20-60 \mathrm{~nm}$ of size and can be produced via polymerization [12]. PANI increases the light absorption property of the perovskite layer by improving the surface of perovskite thin film introducing the nanoparticles into a thin film. Conductive hole transport material in OLED is layered with PANI.

\section{Polytriarylamines (PTAAs)}

An exceptional electron blocking capabilities and low ionization is the key importance for choosing PTAA as a hole transport material in perovskite and OLED research. Amorphous materials have low hole mobility and PTAA has high void space. The crystal structure of PTAA can be improved by introducing planar moieties to transform it into more crystalline HTM material [13]. Hole mobility of the material can be enhanced by suitable doping with cobalt compounds. PTAA containing Hydrophobic alkyl chain has a higher tendency to show hysteresis rather than hydrophilic ones.

\section{Polyfluorenes (PFO)}

Polyfluorenes and polyfluorene doped products are used as hole-transporting materials due to efficient hole extraction in perovskite solar cells. Only PFO has low oxidation potential and low valence band compared to perovskite. Suitable doping and structure change is possible by introducing an electron-rich triarylamine monomer that improves the valence band of the perovskite [14]. After structure modification, it works better than polyfluorenes and better than spiro-OMeTAD in terms of hole extraction ability.

\section{Carbazole}

Due to desired electrical and tunable optical properties conjugated polymer based on Carbazole are now being used in perovskite-based solar cells. As an ideal hole transport material Carbazole based polymers are suitable in perovskite solar cells [15]. By copolymerization process with 3,4-ethylene di-oxythiophene (EDOT), the newly formed compound has more electron-donating ability. 2,7-Cbz-EDOT and 3,6-Cbz-EDOT are the most popular among carbazole based material which is used as a hole transport material in perovskite solar cells.

\section{Polymer-based on sulfur-selenium materials}

Recent research reveals that sulfur-selenium material-based polymer can be used in perovskite solar cells as a hole transport layer. Photoluminescence property and photoinduced absorption quality are ideal for transfer quality charges. Sulphur-selenium based polymeric material used in perovskite solar cell has achieved $10.21 \%$ efficiency [16]. The conductivity of this material is relatively good than other many polymeric materials. By introducing selenium material in the polymeric sulfur, it reduces the optical bandgap of the polymer. Selenium doped sulfur polymer has an impact on photoluminescence property of thin-film perovskite.

\section{Low Band Gap Polymers Application in Perovskite Solar Cell}

Low bandgap polymers are introduced in OPV as well as perovskite solar cells to improve the absorption of infrared or near-infrared region wavelengths of light [17]. Generally, lower bandgap polymer has higher hole transfer mobility than other HTM. Different variables like processing time, temperature, additives used has a high impact on the bandgap of the polymers. Low bandgap polymer HTM like, PCBTDPP, PCDTBT or PCPDTBT has been tested by researchers but any significant improvement was not found from their observation and all these materials are belongs to thiophene based polymers [18]. PTB7 similar type thiophene based polymer works like hole transporting material has reported decent improvement in power output in perovskite solar cell. PTB7 has higher hole mobility than P3HT and even widely used spiro-OMeTAD [19]. PTB-DCB21 polymer has a dichlorobenzene 
functional group exhibit higher power conversion efficiency [19]. It works as a bridge between perovskite and HTM layer. Lower the recombination rates and faster the electron transfer leads to higher efficiency.

\section{A Polymer-perovskite Composite Material in Hybrid Perovskite Devices}

The polymer-perovskite composite used in making perovskitebased LED is termed as heterostructure. This structure is a combination of perovskite components layered with an insulating polymer. Perovskite structure is made with 2D and 3D components. Charge carriers move from 2D to 3D regions and in the 3D regions charges are separated out that lead to recombination and light emission from the perovskite-based LED [20]. The perovskite material and polymers blend most prevent the charge carriers from recombination and prevent the device from non-emissive losses. This hybrid perovskite structure with LED when first developed was facing losses charge carriers from the perovskite layer due to the micro defects in the perovskite crystal structure which leads to lowering their light-emitting efficiency [21,22]. After a lot of development and research by adding polymer with the perovskite material it is now possible to transfer charge faster from 2D to 3D regions and charge extraction from 3D regions in an efficient way.

\section{How Polymers Improve Perovskite Solar Cell Performance?}

Solution processing followed by the heating method introduced defects at grain boundaries of the perovskite thin-film layer. A suitable number of additives adding not only changes the surface morphology of thin film but also increases the stability of the perovskite material in challenging ambient conditions. Suitable tuning in grain boundary morphology helps to improve the optoelectronic property of the solar cell as well as increases the stability of the cell [23]. When polymer additives (some specific polymer) are added to the perovskite material, polymer and perovskite molecules form a cross-linked polymer chain like substance. These cross-link's materials help the perovskite material to become more stable in humid ambiance and protect from electrical decoupling between two adjacent grains [24].

Adding surfactants having a smaller molecule has a significant impact on the power conversion efficiency of the perovskite solar cell in solution processing methods. It was observed that Suitable polymer addition with perovskite changes the carrier dynamics and the surface morphology. Recent research reveals that using 4-vinyl pyridine (PVP) with Methylammonium lead iodide (MAPbI3) has a large improvement in the open-circuit voltage of the fabricated solar cell, consequently, the cell's performance-enhanced significantly [25]. PVP not only enhances the power output but also addressed the degradation issue of the cell. Perovskite solar cells coated with a thin-film plasma polymer layer become more stable in humid conditions. This encapsulation helps the cell to prevent degradation under moisture and humid weather. This thin-film polymer is deposited on the perovskite layer by the plasma vacuum deposition method and performed at room temperature [26]. The absorbance quality of the perovskite layer does not have an impact after polymer film deposition and very less impact on power conversion efficiency.

\section{Some Drawbacks of Polymers Used in Perovskite Solar Cell}

The transparent polymer layer on perovskite provides better stability and passivation. Apart from the many advantages, polymers have a few drawbacks in perovskite solar cell applications. Thermal stability is one of the most important issues for polymers in the perovskite solar cell application, apart from this, the ability of water retention and adhesive forces characteristics of the polymer largely has an impact on the performance of the perovskite solar cell [27].

The thermal stability of a polymer encapsulated perovskite solar cell in high ambient temperature conditions is the main concern and opens new challenges for further research. A significant amount of thermal stress evolved in the solar cell during the high-temperature ambient condition and that leads to thermal instability and material degradation. Thermal stress and followed by thermal degradation can be encountered by introducing a lower bandgap donor polymer which is efficient in harvesting near-infrared region light [28].

The adhesive property of thin-film solar cells encapsulated with polymer substance highly depends on solution processing and thermal treatment during fabrication. The adhesive property of the substance largely influences the multi-layered device structure [29]. Adhesive property change may raise the strains in the perovskite solar cell layer which may be caused by many reasons like environmental conditions, handling the material wrongfully, inhomogeneous thermal expansion or shrinkage formation during the processing of solution [30].

Perovskite material is significantly affected by water molecules $[31,32]$. To improve the stability of perovskite solar cells we must prevent water penetration. NiO (Nickel oxide), Silicon dioxide (SiO2), etc hydrophilic oxide molecules help the perovskite layer from water retention. Dual Parylene transparent polymer layer improved the passivation effects and helps from water retention by strong molecular binding of Parylene with SiO2 [33].

\section{Conclusion}

Despite having high-performance efficiency in perovskite solar modules are still not commercialized. Instability is one of the reasons. Adding polymers to lead-based halide perovskite leads to increasing chemical stability. In another way, the addition of polymers in perovskite solar cells provides various solutions like quality charge transport and works as an absorber. Polymers are widely used in PSC which regulates the crystallization of perovskite films that leads to enhances cell performance and stability. Due to their high hole mobility, these polymers can be employed as the hole transfer materials also. Thermal stability in high ambient conditions, the ability of water retention, and adhesion property still an issue for polymer application in perovskite solar cells, and further research is going on to optimize the performances and counter the issues. 


\section{Acknowledgment}

This work was supported by Science and Engineering Research Board (SERB) grants funded by Department of Science and Technology (DST) Central, Government of India through Teachers Associateship for Research Excellence (TAR/2018/000195).

\section{References}

1. Hou W, Xiao Y, Han G, Lin JY (2019) The applications of polymers in solar cells: A review. Polymers 11(1): 143.

2. Müller K, Bugnicourt E, Latorre M, Jorda M, Echegoyen Sanz Y, et al. (2017) Review on the processing and properties of polymer nanocomposites and nano-coatings and their applications in the packaging, automotive and solar energy fields. Nanomaterials 7(4): 74.

3. Roy S, Kargupta K, Chakraborty S, Ganguly S (2008) Preparation of polyaniline nanofibers and nanoparticles via simultaneous doping and electro-deposition. Materials Letters 62(16): 2535-2538.

4. Kim H, Lee J, Kim B, Byun HR, Kim SH, et al. (2019) Enhanced stability of MAPbI3 perovskite solar cells using Poly(p-chloro-xylylene) encapsulation. Scientific Reports 9(1): 15461.

5. Zhao X, Kim HS, Seo JY, Park NG (2017) Effect of selective contacts on the thermal stability of perovskite solar cells. ACS Applied Materials \& Interfaces 9(8): 7148-7153.

6. Mabrouk S, Bahrami B, Elbohy H, Reza KM, Gurung A, et al. (2019) Synergistic engineering of hole transport materials in perovskite solar cells. InfoMat.

7. Zhang L, Wu J, Li D, Li W, Meng Q, et al. (2019) Ladder-like conjugated polymers used as hole-transporting materials for high-efficiency perovskite solar cells. Journal of Materials Chemistry A 7(24): 1447314477.

8. Azzouzi M, Kirchartz T, Nelson J (2019) Factors controlling open-circuit voltage losses in organic solar cells. Trends in Chemistry 1(1): 49-62.

9. Luzio A, Criante L, D Innocenzo V, Caironi M (2013) Control of charge transport in a semiconducting copolymer by solvent-induced long-range order. Scientific Reports 3: 3425.

10. Ahmed EM (2015) Hydrogel: Preparation, characterization, and applications: A review. Journal of Advanced Research 6(2): 105-121.

11. Fairfield DJ, Sai H, Narayanan A, Passarelli JV, Chen M, et al. (2019) Structure and chemical stability in perovskite-polymer hybrid photovoltaic materials. Journal of Materials Chemistry A 7(4): 16871699.

12. Wang X, Ye KH, Yu X, Zhu J, Zhu Y, et al. (2018) Polyaniline as a new type of hole-transporting material to significantly increase the solar water splitting performance of $\mathrm{BiVO}_{4}$ photoanodes. Journal of Power Sources 391: $34-40$

13. Liu Y, Liu Z, Lee EC (2019) High-performance inverted perovskite solar cells using doped Poly(triarylamine) as the hole transport layer. ACS Applied Energy Materials 2(3): 1932-1942.

14. Zhang L, Wu J, Li D, Li W, Meng Q, et al. (2019) Ladder-like conjugated polymers used as hole-transporting materials for high-efficiency perovskite solar cells. Journal of Materials Chemistry A 7: 14473-14477.

15. Li W, Otsuka M, Kato T, Wang Y, Mori T, et al. (2016) 3,6-Carbazole vs 2,7-carbazole: A comparative study of hole-transporting polymeric materials for inorganic-organic hybrid perovskite solar cells. Beilstein Journal of Organic Chemistry 12: 1401-1409.

16. Liu P, Kloo L, Gardner JM (2017) Cross-linked sulfur-selenium polymers as hole-transporting materials in dye-sensitized solar cells and perovskite solar cells. Chem Photo Chem 1(8): 363-368.
17. Sun C, Xia R, Shi H, Yao H, Liu X, et al. (2018) Heat-insulating multifunctional semitransparent polymer solar cells. Joule 2(9): 18161826.

18. Peng SH, Huang TW, Gollavelli G, Hsu CS (2017) Thiophene and diketopyrrolopyrrole based conjugated polymers as efficient alternatives to spiro-OMeTAD in perovskite solar cells as hole transporting layers. Journal of Materials Chemistry C 5: 5193-5198.

19. Lee JW, Park S, Ko MJ, Son HJ, Park NG (2014) Enhancement of the photovoltaic performance of $\mathrm{CH}_{3} \mathrm{NH}_{3} \mathrm{PbI}_{3}$ perovskite solar cells through a dichlorobenzene-functionalized hole-transporting material. Chem Phys Chem 15(12): 2595-2603.

20. Motti SG, Crothers T, Yang R, Cao Y, Li R, et al. (2019) Heterogeneous photon recycling and charge diffusion enhance charge transport in Quasi-2D lead-halide perovskite films. Nano Letters 19(6): 3953-3960.

21. Vidyasagar CC, Muñoz Flores BM, Jiménez Pérez VM (2018) Recent advances in synthesis and properties of hybrid halide perovskites for photovoltaics. Nano-Micro Letters 10: 68

22. Moyez SA, Roy S (2017) Thermal engineering of lead-free nanostructured $\mathrm{CH}_{3} \mathrm{NH}_{3} \mathrm{SnCl}_{3}$ perovskite material for thin-film solar cell. Journal of Nanoparticle Research 20: 5.

23. Chen K, Schünemann S, Song S, Tüysüz H (2018) Structural effects on optoelectronic properties of halide perovskites. Chemical Society Reviews 47: 7045-7077.

24. Wei D, Huang H, Cui P, Ji J, Dou S, et al. (2019) Moisture-tolerant super molecule for the stability enhancement of organic-inorganic perovskite solar cells in ambient air. Nanoscale 11: 1228-1235.

25. Yavari M, Mazloum-Ardakani M, Gholipour S, Tavakoli MM, Taghavinia N, et al. (2018) Reducing surface recombination by a Poly(4-vinylpyridine) interlayer in perovskite solar cells with high open-circuit voltage and efficiency. ACS Omega 3(5): 5038-5043.

26. Swartwout R, Hoerantner MT, Bulović V (2019) Scalable deposition methods for large-area production of perovskite thin films. Energy \& Environmental Materials 2(2): 119-145.

27. Pitchaiya S, Natarajan M, Santhanam A, Asokan V, Yuvapragasam A, et al. (2018) A review on the classification of organic/inorganic/carbonaceous hole transporting materials for perovskite solar cell application. Arabian Journal of Chemistry 13(1): 2526-2557.

28. Wang L, Peng M, Li X, Wang Y, Luo H, et al. (2019) Suppressing the thermal degradation of bismuth near-infrared luminescence in optical amorphous materials via topologically polymerized network structures. Journal of Materials Chemistry C 7: 5074-5083.

29. Qiu L, Ono LK, Qi Y (2018) Advances and challenges to the commercialization of organic-inorganic halide perovskite solar cell technology. Materials Today Energy 7: 169-189.

30. Lin Y, Fang Y, Zhao J, Shao Y, Stuard SJ, et al. (2019) Unveiling the operation mechanism of layered perovskite solar cells. Nature Communications 10(1): 1008.

31. Huang J, Tan S, Lund PD, Zhou H (2017) Impact of $\mathrm{H}_{2} \mathrm{O}$ on organicinorganic hybrid perovskite solar cells. Energy \& Environmental Science 10: 2284-2311.

32. Moyez SA, Roy S (2018) Dual-step thermal engineering technique: A new approach for fabrication of efficient $\mathrm{CH}_{3} \mathrm{NH}_{3} \mathrm{PbI}_{3}$-based perovskite solar cell in open air condition. Solar Energy Materials and Solar Cells 185: 145 .

33. Park DY, Byun HR, Kim H, Kim B, Jeong MS (2018) Enhanced stability of perovskite solar cells using organosilane-treated double polymer passivation layers. Journal of the Korean Physical Society 73: 1787-1793.

For possible submissions Click below: 